\title{
DESCRIÇÃO DO PERFIL SOCIOECONÔMICO DA AQUICULTURA NO ESTADO DO ESPÍRITO SANTO
}

\author{
Julianne Almeida Rodrigues ${ }^{1}$ \\ Winny Silva Trugilho ${ }^{2}$ \\ Bruno Fardim Christo ${ }^{3}$ \\ Fernanda Dassie Rangel ${ }^{4}$ \\ Elaine Cristina Gomes da Silva ${ }^{5}$
}

Resumo: O Estado do Espírito Santo possui uma economia fundamentalmente agrícola. Sua riqueza hídrica aliada às temperaturas altas durante todo o ano, potencializam a região para o desenvolvimento da aquicultura tropical. O objetivo deste trabalho é descrever o perfil da aquicultura no Estado do Espírito Santo, apontando a importância para o desenvolvimento econômico e social do estado, através de uma pesquisa exploratória, descritiva e qualitativa. Ao término deste trabalho pôde-se concluir que o estado apresenta condições que favorecem o desenvolvimento da atividade que se encontra ainda, muito familiar, pouco tecnificada e com níveis de produtividade muito baixos.

Palavras-chave: Aquicultura; Desenvolvimento; Espírito Santo.

\footnotetext{
${ }^{1}$ Zootecnia/Universidade Federal do Espírito Santo, Brasil. E-mail: julianne_ar@hotmail.com.

2 Mestrado em Produção Vegetal/Universidade Estatual do Norte Fluminense Darcy Ribeiro, Brasil. E-mail: winnytrugilio@hotmail.com.

${ }^{3}$ Agronomia/Universidade Federal do Espírito Santo, Brasil. E-mail: brunochristo@hotmail.com.

${ }^{4}$ Eng. Química/Universidade Federal do Espírito Santo, Brasil. E-mail: fernanda.dassierangel@gmail.com.

${ }^{5}$ Ciências Florestais/Universidade Federal do Espírito Santo, Brasil. E-mail: ecristinags@gmail.com.
} 